\title{
Drone Based Wireless Mesh Network for Disaster/Military Environment
}

\author{
Gudi Siva Leela Krishna Chand1, Manhee Lee ${ }^{2}$, Soo Young Shin ${ }^{2 *}$ \\ ${ }^{1}$ Innovation and Enterprise Research Lab., University of Technology, Sydney, Australia \\ ${ }^{2}$ Dept. of IT Convergence Engineering, Kumoh National Institute of Technology, Gumi, Gyeoungbuk, Republic of Korea \\ Email: leelakrishnachand@gmail.com,1mh910420@gmail.com, *wdragon@kumoh.ac.kr
}

How to cite this paper: Chand, G.S.L.K., Lee, M. and Shin, S.Y. (2018) Drone Based Wireless Mesh Network for Disaster/Military Environment. Journal of Computer and Communications, 6, 44-52. https://doi.org/10.4236/jcc.2018.64004

Received: March 18, 2018

Accepted: April 23, 2018

Published: April 26, 2018

Copyright ( 92018 by authors and Scientific Research Publishing Inc. This work is licensed under the Creative Commons Attribution International License (CC BY 4.0).

http://creativecommons.org/licenses/by/4.0/

(c) (i) Open Access

\begin{abstract}
In recent years, researches of disseminating wireless network have been conducted for areas without network infrastructure such as disaster situation or military disputes. However, conventional method was to provide a communication infrastructure by floating large aircraft as UAV or hot-air balloon in the high air. Therefore, it was difficult to utilize previous method because it requires a lot of time and cost. But it is possible to save money and time by using a drone which is already used in many areas as a small UAV. In this paper, we design a drone that can provide wireless infrastructure using high speed Wi-Fi. After reaching the target area, the drone can provide Wi-Fi using wireless mesh network and transmit the situation of local area through attached camera. And the transmitted videos can be monitored in the control center or cell phone through application in real time. The proposed scheme provides wireless communication of up to $160 \mathrm{Mbps}$ in a coverage of about $200 \mathrm{~m}$ and video transmission with a coverage of about $120 \mathrm{~m}$, respectively.
\end{abstract}

\section{Keywords}

Drone, Wireless Mesh Network (WMN), Wi-Fi

\section{Introduction}

Recently, natural disaster like earthquake has occurred in the local, and there is a risk of collapse of buildings due to terrorism or disputes around the world [1]. In addition, there is an area where even the cellular network cannot be used in a situation where the existing communication infrastructure is destroyed or reinstallation is difficult. The absence of short-term communication can be fatal impact because communication is readily available everywhere in the modern ${ }^{*}$ Corresponding author. 
world [2]. For example, communication systems were cut off due to power outage for several days in the Pacific coast which was happened from 2011 East Asian earthquake. The situation where about 29,000 base stations and 1.9 million wired communication systems were broken makes additional damages owing to lack of spreading news [3]. In 2012, many infrastructures were destroyed by Hurricane Sandy, the largest ever North Atlantic in the United States but using social media such as Twitter has been able to spread the situation and reduce additional corruptions through informational alerts [4].

Researched about supporting wireless Internet in the areas where there is no communication infrastructure has been investigated in various companies. The way Facebook suggests is to make an airplane of similar size to the Boeing 747 float up high in the air [5]. Google is using large aircraft, but it has characterized by advanced antenna with very high frequency (VHF) [6]. However, in order to provide continuous wireless Internet, it should be able to fly for a long time, and there should be problems on the weather or airspace and it causes cost a lot because of serving a large area. In particular, disaster and dispute areas need a way to utilize existing communication infrastructure and maintain short-term communication.

The drone, which has been developed for military use and has a smaller unmanned air vehicle (UAV), can be solution for disaster and dispute areas. It has been used throughout the pre-sale of broadcasting such as broadcasting relay, weather observation and unmanned security [7]. The battery is mainly used as a power source which affects flight time and payload. It is also easy to be replaced, manufacture and has the advantage of low cost. Furthermore, it can be smaller than large aircraft so that it can spread to various regions.

There are diverse methods such Bluetooth, cellular, and Wi-Fi for establishing a communication infrastructure using a drone. The characteristics vary depending on each method. In the case of Bluetooth, it is mainly used when low power is needed in a short distance. By frequency hopping technology, interference problem is reduced. But, it is difficult to transmit high-capacity of data such as pictures or videos since the transmission speed is slower than that of Wi-Fi. In the case of cellular, it provides communication service over a wide area based on the base station and has the advantage that handover problem is the least. However, it is hard to use the cellular network in the sky and there is a quality problem when less base stations are located. Finally, in the case of Wi-Fi, interference between devices using the same channel may occur but it is possible to transmit data with high-speed and can be easily accessed through a general laptop or smart phone without extra receiver. Also, the distance of communication can be increased through the network constructing wireless mesh topology [8]. A wireless mesh network is a technology using wireless LAN that enables a low-cost and efficient network to be established where a network infrastructure is not built. Mesh nodes are connected to each other, and each node is a host and router. Unlike other wireless networks, it is possible to increase the communication 
capacity according to the number of nodes and hops, and it is emerged as a promising technology for broadband Internet because it uses hardware based on IEEE 802.11 [9].

In this paper, we design a drone that provides high-speed $\mathrm{Wi}-\mathrm{Fi}$ by constructing a wireless mesh network. It will be dispatched into disaster/dispute areas in order to avoid disturbance propagation and to spread information. The drone was selected as quad-copter to be capable of stable and easy flight control. Also, it can be compatible with existing communication equipment with mesh node which is based on IEEE 802.11 to provide high-speed Wi-Fi. Efficient wireless mesh network is organized with BATMAN-advanced based MAC address working on Layer 2 [10].

This paper is composed as follows. Section 2 addresses that the proposed system model and shows structure of the implemented drone by pictures. It also specifies the implementation of the test bed and equipment in detail. In Section 3 performance evaluations are described by measurement such as maximum distance, real-time image transmission, and flight time of the drone. This paper is concluded in Section 4.

\section{Drone-Based Wireless Mesh Network}

\subsection{System Model}

In this section, we propose a drone that can configure a wireless mesh network to support the areas where network infrastructure is destroyed by disasters or disputes. A scenario is shown in Figure 1 perceptually to provide wireless network with hopping devices as drones. In this scenario, the base station dispatches a number of proposed drones to the area and drones are distributed at regular intervals when these arrive at the destination. By searching nearby drones to establish wireless mesh network automatically, it provides high-speed Wi-Fi to the area. Using the camera installed in each drone, situation of the area can be transmitted to the control center in real time. It is possible to make efficient network checking the data capacity of each node i.e. drone and adjusting the arrangement of drones corresponding to the region where the capacity limit is exceeded.

\subsection{Wireless Mesh Network}

We design a wireless mesh network using OM2P and MR1750 mesh nodes of Open-Mesh as shown in Figure 2. OM2P is a node supporting IEEE 802.11n with $2.4 \mathrm{GHz}$ frequency band and MR1750 is a node supporting IEEE 802.11ac with $5 \mathrm{GHz}$ frequency bands. Each mesh nod has a function to act as a gateway depending on whether wired LAN (WLAN) is used or not.

The operating system of mesh node uses Open WRT which is an open source based on Linux [11]. For exploiting BATMAN-advanced, BATMAN kernel module is installed on each mesh node, shell programming script is written, and each mesh node is automatically connected to form a wireless mesh network. The used BATMAN script is run according to the flowchart shown in Figure 3. 


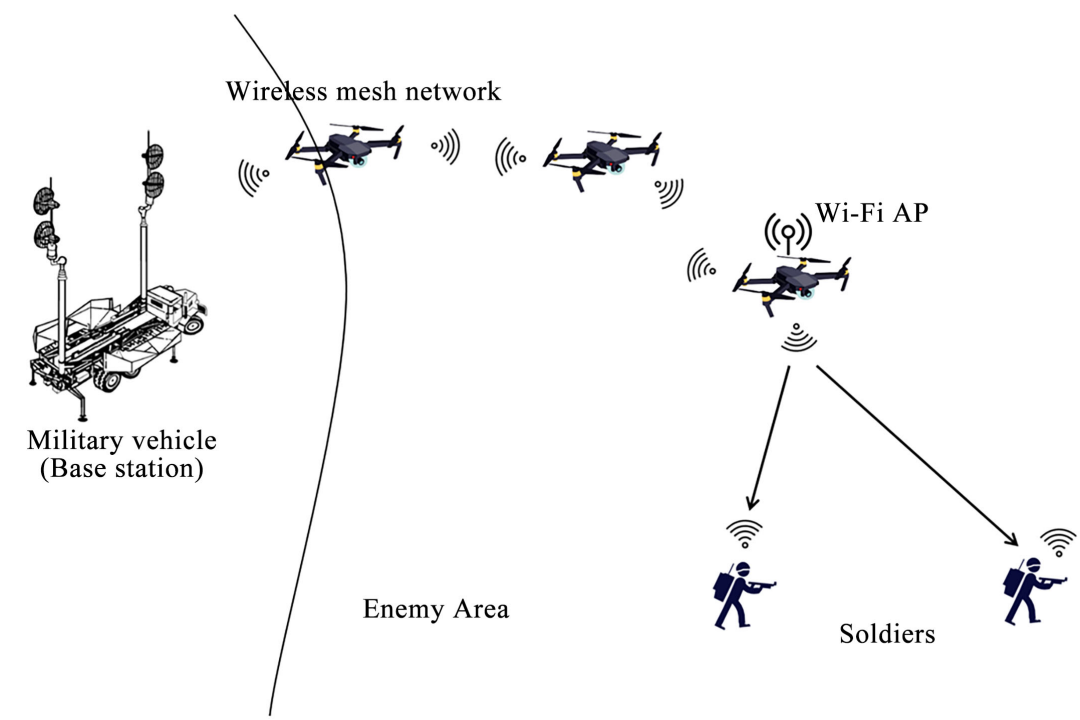

Figure 1. Secnario of the proposed system. (Military environment).
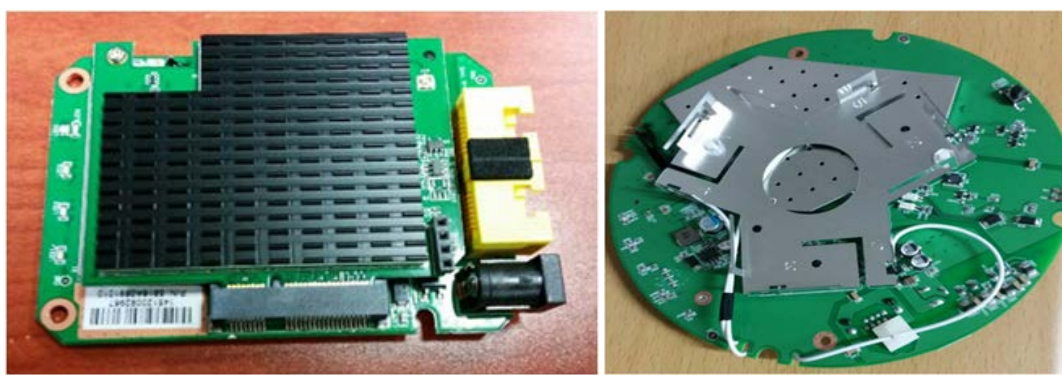

Figure 2. Wireless mesh network nodes (left) OM2P, (right) MR1750.

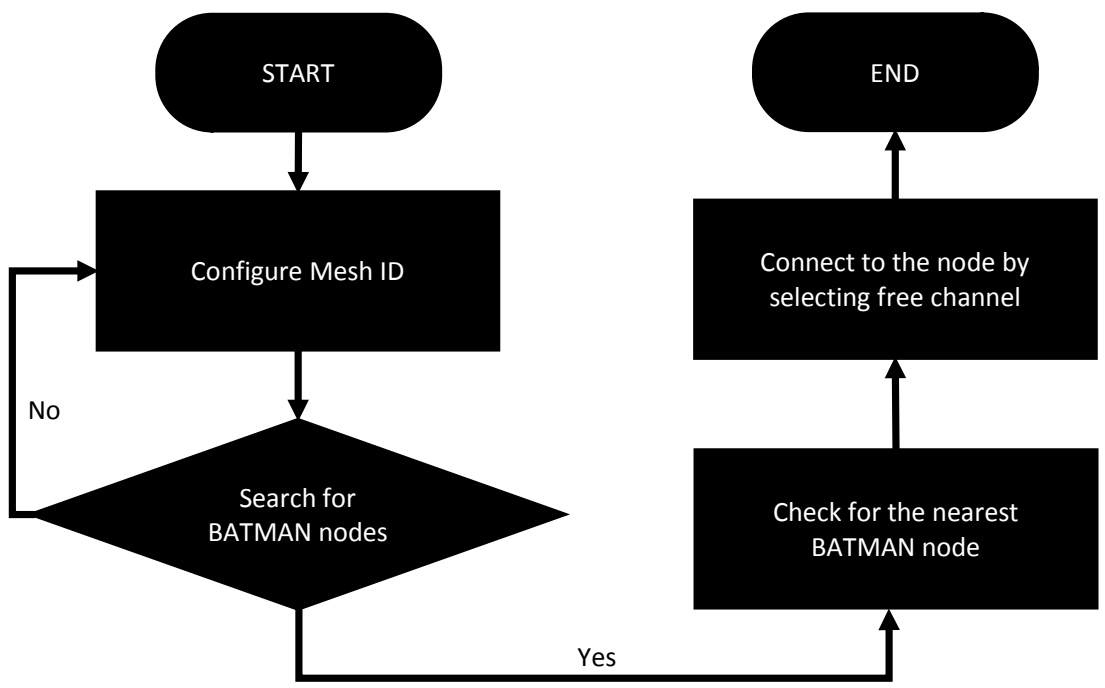

Figure 3. Flowchart of BATMAN-advanced script.

Each mesh node is defined to have the same service set identifier (SSID) for easy group assignment. These nodes search for adjacent nodes and confirm to make mesh network. 
As shown in Figure 4, wireless mesh network with BATMAN-advanced is consisted of three access points (Aps) and a cell phone. Among mesh nodes, one is connected with a WLAN so as to test Internet access. The nodes are arranged at regular intervals and they are automatically scanned to confirm the use of Internet.

\subsection{Drone with Wireless Mesh Node}

Drone has been developed for military purposes and become popular as open source being used in various fields such as law enforcement, agriculture, entertainment, disaster recovery, mining, etc. [12]. Previously, drone is used only as a surveillance role for the delivery of goods for specific purposes but it is possible to utilize the drone as a search tool activating the automatic flight with exact coordinates. In general, drone flies low it can be advantageous to supply Internet using wireless mesh node. And it is suitable for use in dangerous areas because of remote control.

We use a quad-copter that is stable and easy to control flight. Unlike general drone, we consider a payload to fly with a mesh node to support wireless network and a camera for real time image transmission. The total weight of the parts is calculated to be $2.3 \mathrm{~kg}$, which is theoretically to consume 17 A per hour. It can fly about 20 minutes with $6000 \mathrm{mAh}$ battery. If we use higher capacity battery, flight time get readily longer. It is consisted of flight controller (FC), electronic speed control (ESC), motors, propellers and a GPS. A camera for capturing images is located on the front of proposed drone and a mesh node is installed on bottom of proposed drone. Figure 5 shows each major part separately. This drone can support Wi-Fi by using the mounted mesh node, alert the current coordinate information through GPS and transmit situation of the area deployed through the camera.

\section{Performance Evaluation}

In this section, we evaluate the transmission distance and speed of Wi-Fi to compare performance of proposed drone. The pixel and frame rate of the image are designated to evaluate maximum distance for real time transmission. Finally, flight time is calculated to check mobility of the drone. Drone, R2 is connected to $\mathrm{R} 1$ by one hop at a distance of about $30 \mathrm{~m}$.

\subsection{Transmission Distance}

To test transmission distance for two purposes, two mesh nodes are set up with scenarios as shown in Figure 6 and denoted R1 and R2, respectively. R1 is located in an indoor room, serves as a base station and connected into the IP camera at the same location. In the outdoor, we use a smart phone to connect with wireless mesh drone, R2, and confirm distance between IP camera. The maximum distance is checked by application. Figure 7 shows the test result in six different environments at various locations. 


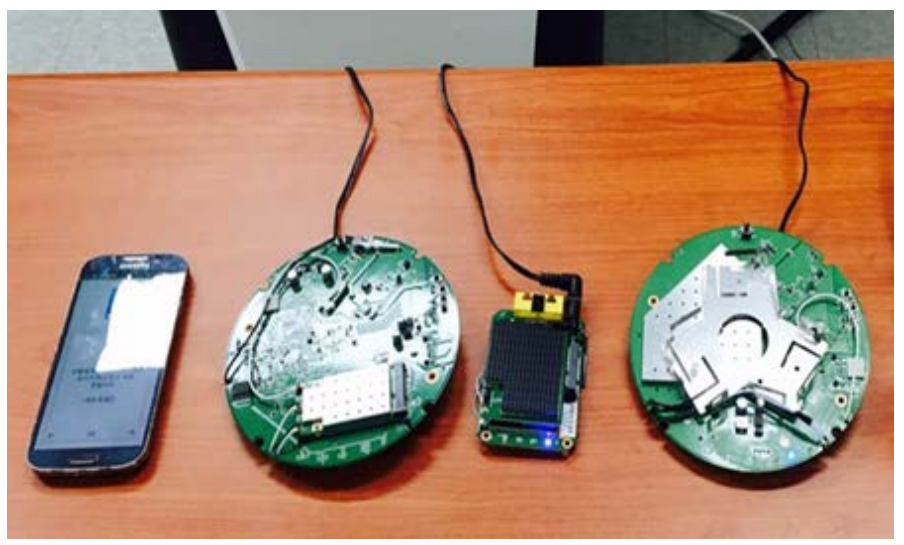

Figure 4. Wireless mesh network communication test.

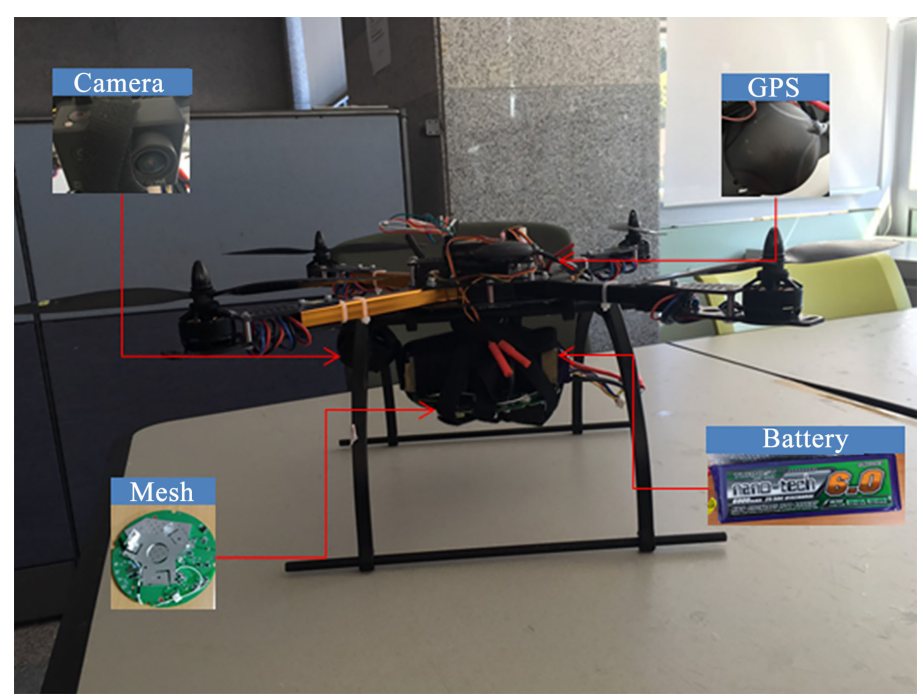

Figure 5. Implemented drone with wireless mesh network.

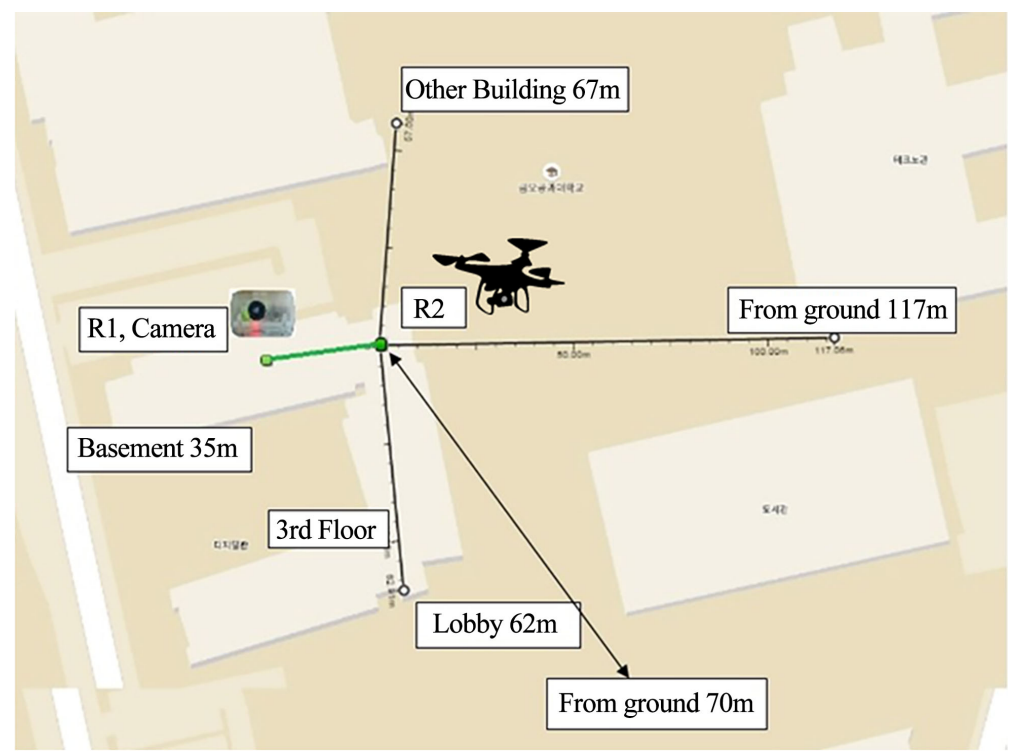

Figure 6. Performance test with wireless mesh network with drone for video and Internet access. 


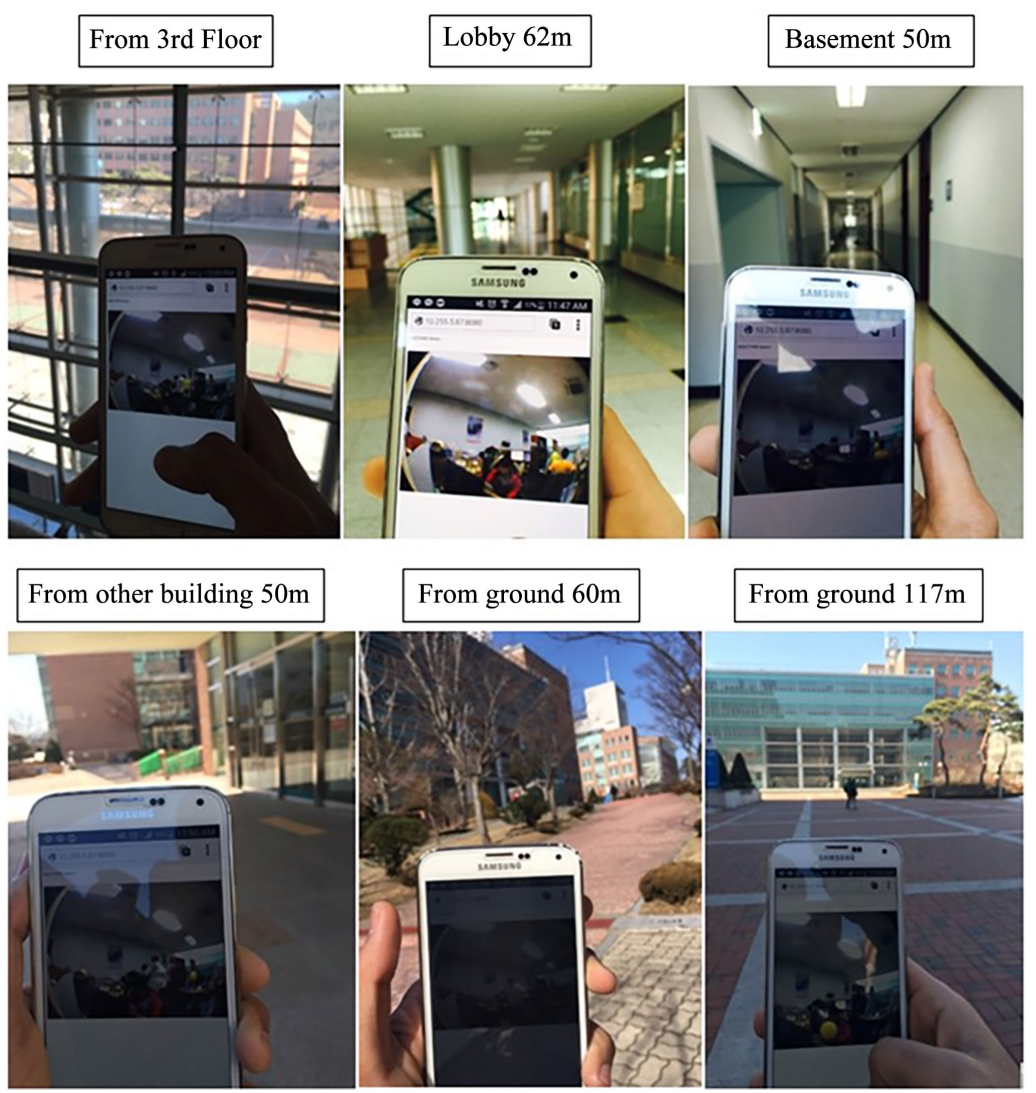

Figure 7. Test snap shots for video and Internet access.

First purpose is on real time video transmission. OM2P (802.11n) node is possible up to $60 \mathrm{~m}$ and MR1750 (802.11ac) node is up to $120 \mathrm{~m}$. In the second purpose is on Internet connection. 802.11n node is able to access the Internet up to $100 \mathrm{~m}$ and $802.11 \mathrm{ac}$ node is up to $200 \mathrm{~m}$. Test results show that distance of Internet connection is improved when proposal drone uses $\mathrm{R} 2$ as a node. It is presented as about $30 \mathrm{~m}$ for $802.11 \mathrm{n}$ node and $75 \mathrm{~m}$ for $802.11 \mathrm{ac}$ node. This is because the characteristic of line-of-sight (LOS) is applied by proposed drone.

\subsection{Transmission Speed}

To measure the transmission rate of each node, open source software Iperf is used [13]. The measurement results show that transmission speed of $802.11 \mathrm{n}$ node is up to $80 \mathrm{Mbps}$ and that of $802.11 \mathrm{ac}$ node is up to $160 \mathrm{Mbps}$ in line-of-sight. It is identified that the transmission speed is improved up to 10 Mbps for $802.11 \mathrm{n}$ and up to $15 \mathrm{Mbps}$ for 802.11ac node with non-line-of-sight (NLOS). Figure 8 shows a snapshot of the transmission speed test.

Finally, for measuring the operation time of implemented drone, a wireless mesh node is activated/connected with drone via wireless mesh network and the drone is connected to Internet via R1. And the drone is set to maintain at an altitude of $100 \mathrm{~m}$. With full battery, it is able to fly for about 25 minutes and it is tested by 20 times with same drone and same configuration. Table 1 summarizes overall evaluation. 


\begin{tabular}{|c|c|c|c|c|c|c|c|c|}
\hline \multicolumn{2}{|c|}{ Access Points $\mathbf{A}$} & Network Map & All Networks Map & \multirow{2}{*}{$\begin{array}{l}\text { Clients } \\
\text { Last Checkin }\end{array}$} & \multirow{2}{*}{$\begin{array}{l}\text { Site Survey } \\
\text { Up time }\end{array}$} & \multicolumn{2}{|c|}{ Network Diagram } & \multirow[b]{2}{*}{$\begin{array}{l}\text { Mesh Speed } \\
\text { (Now/Avg) }\end{array}$} \\
\hline Status $^{\wedge}$ & Name/Notes & Clients & Usage $\uparrow$ (MB) & & & Hops & $\begin{array}{l}\text { Nearest } \\
\text { Gateway }\end{array}$ & \\
\hline 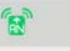 & $\frac{R 1-5 P}{o m 5 P}$ & 0 & $\frac{O B}{(10 B, 10 B)}$ & $2 \mathrm{~m}: 51 \mathrm{~s}$ & $38 \mathrm{~m}$ & 1 & $\begin{array}{l}\text { R2--MR } \\
5.25 .212 .32\end{array}$ & $\begin{array}{l}{ }_{157.8 \mathrm{Mbps}} \\
130.7 \mathrm{Mbps}\end{array}$ \\
\hline 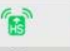 & $\underline{\mathrm{R} 3-2 \mathrm{P}}$ & 4 & $\frac{529 \mathrm{MB}}{(1407.3 \mathrm{MB},+121.8 \mathrm{MB})}$ & $3 \mathrm{~m}: 17 \mathrm{~s}$ & $1 \mathrm{~h} 47 \mathrm{~m}$ & 1 & $\begin{array}{l}\text { R2--MR } \\
5.25 .212 .32\end{array}$ & $\begin{array}{l}\square \text { 56.9mbps } \\
\square 9.5 \mathrm{Mbps}\end{array}$ \\
\hline (ब) & MR900new & 0 & $\frac{O B}{(10 B, 1 O B)}$ & $57 \mathrm{~m}: 15 \mathrm{~s}$ & Down & 0 & $\begin{array}{l}\text { self } / 172.16 .1 .4 \\
202.31 .137 .54\end{array}$ & $\begin{array}{l}\text { N/A } \\
\text { N/A }\end{array}$ \\
\hline (⿶) & $\underline{\mathrm{R} 2-\cdots \mathrm{MR}}$ & 5 & $\frac{4.8 \mathrm{MB}}{(13 \mathrm{MB},(1.8 \mathrm{MB})}$ & $1 \mathrm{~m}: 46 \mathrm{~s}$ & $20 \mathrm{~d} 17 \mathrm{~h} 30 \mathrm{~m}$ & 0 & $\begin{array}{l}\text { self/ } / 172.16 \cdot 1.5 \\
202.31 .137 .54\end{array}$ & $\begin{array}{l}\mathrm{N} / \mathrm{A} \\
\mathrm{N} / \mathrm{A}\end{array}$ \\
\hline
\end{tabular}

Figure 8. Snap shot of transmission speed test.

Table 1. Table type styles (Table caption is indispensable).

\begin{tabular}{ccc}
\hline Item & Unit & Value \\
\hline Distance for transmission & $\mathrm{m}$ & $\sim 100(802.11 \mathrm{n})$ \\
& & $\sim 200(802.11 \mathrm{ac})$ \\
Transmission speed & Mbps & $\sim 80(802.11 \mathrm{n})$ \\
Resolution of video & Pixels & $160(802.11 \mathrm{ac})$ \\
Frame rate of video & Frame per second & 29 \\
Flight time of drone & minitues & 25 \\
\hline
\end{tabular}

\section{Conclusions}

Many researches have been inquired to disseminate wireless Internet in areas where network infrastructure is no sufficient such as disaster or dispute areas. However, conventional method was to float a large air vehicle in the sky and it was not suitable for emergency situations because it required a lot of time and money. In this paper, we propose a drone which uses wireless mesh network nodes as a way to reduce cost and save time. It is possible to provide instant wireless communication infrastructure with high speed Wi-Fi. The drone is dispatched to the target area by automatic flight mode and the BATMAN-advanced based mesh network is used to provide Internet access. Through a camera, local situation can be transmitted in real time. The proposed drone has a flight time of about 25 minutes, can provide up to $80 \mathrm{~m}$ bps transmission speed at a distance of $100 \mathrm{~m}$ and video transmission in real time at a distance of $60 \mathrm{~m}$.

In the future, it will be developed using an inflatable balloon with flexible solar panel as a method for extending flight time and network operating time. Based on low-power wide-area network (LPWAN) communication, coordinates can be easily used for automatic flight information.

\section{Acknowledgements}

This research was supported by the MSIT (Ministry of Science and ICT), Korea, under the ITRC (Information Technology Research Center) support program (IITP-2017-2014-0-00639) supervised by the IITP (Institute for Information \& communications Technology Promotion).

\section{References}

[1] Sheen, D.-H. (2015) Comparison of Local Magnitude Scales in South Korea. Journal 
of the Geological Society of Korea, 51, 415-424.

https://doi.org/10.14770/jgsk.2015.51.4.415

[2] Premkumar, R. (2014) Wireless Networks for Disaster Relief. Technical Report, Washington University, St. Louis.

[3] Masaru, F. (2011) ICT Responses to the Great East Japan Earthquake. http://www.soumu.go.jp/main_sosiki/joho_tsusin/eng/presentation/pdf/111208_1.p df

[4] Wang, H.Y., Hovy, E. and Dredze, M. (2015) The Hurricane Sandy Twitter Corpus. Proceedings of the World Wide Web and Public Health Intelligence, 20-24.

[5] Hern, A. (2015) The Facebook Launches Aquila Solar-Powered Drone for Internet Access. The Guardian.

https://www.theguardian.com/technology/2015/jul/31/facebook-finishes-aquila-sola r-powered-internet-drone-with-span-of-a-boeing-737

[6] John, G. (2015) Welcome to the Space Jam: How United States Regulators Should Govern Google and Facebook's New Internet-Providing High Altitude Platforms. American University Business Law Review, 4, 471-503.

[7] Matolak, D.W. (2015) Unmanned Aerial Vehicles: Communications Challenges and Future Aerial Networking. International Conference on Computing, Networking and Communications (ICNC), 16-19 February 2015, Garden Grove, CA, 567-572. https://doi.org/10.1109/ICCNC.2015.7069407

[8] Zeng, Y., Zhang, R. and Lim, T.J. (2016) Wireless Communications with Unmanned Aerial Vehicles: Opportunities and Challenges. IEEE Communications Magazine, 54, 36-42. https://doi.org/10.1109/MCOM.2016.7470933

[9] Akyildiz, I.F. and Wang, X.D. (2005) A Survey on Wireless Mesh Networks. IEEE Communications Magazine, 43, S23-S30.

https://doi.org/10.1109/MCOM.2005.1509968

[10] Daniel, S., König, A. and Hollick, M. (2011) Routing Performance of Wireless Mesh Networks: A Practical Evaluation of BATMAN Advanced. IEEE 36th Conference on Local Computer Networks (LCN), 897-904.

[11] Palazzi, C.E., Brunati, M. and Roccetti, M. (2010) An OpenWRT Solution for Future Wireless Homes. IEEE International Conference on Multimedia and Expo (ICME), 19-23 July 2010, Suntec City, 1701-1706. https://doi.org/10.1109/ICME.2010.5583223

[12] Marc, C. (2014) Survey of Low Altitude Unmanned Aerial Vehicles. Technical Report, Washington University, St. Louis.

[13] Iperf: The Ultimate Speed Test Tool for TCP, UDP and SCTP Test the Limits of Your Network + Internet Neutrality Test. https://iperf.fr/ 